\begin{abstract}
Annual Report
Advanced Demonstration of Motion Correction for Ship-to-Ship Passive Inspections
\end{abstract}

O R12-Ship2Ship-PD2Jh

Lab:

Project PI:

Primary Author:

Contributors:

Date:

ORNL

Klaus Ziock

Klaus Ziock

Chris Boehnen, Joseph Ernst

9/31/13

Approved for public release.

Distribution is unlimited. 


\section{Advanced Demonstration of Motion Correction for Ship-to-Ship Passive Inspections \\ OR12-Ship2Ship-PD2Jh}

\section{INTRODUCTION}

Passive radiation detection is a key tool for detecting illicit nuclear materials. In maritime applications it is most effective against small vessels where attenuation is of less concern. Passive imaging provides:

- discrimination between localized (threat) and distributed (non-threat) sources,

- removal of background fluctuations due to nearby shorelines and structures,

- source localization to an individual craft in crowded waters, and

- background subtracted spectra.

Unfortunately, imaging methods cannot be easily applied in ship-to-ship inspections because relative motion of the vessels blurs the results over many pixels, significantly reducing sensitivity. This is particularly true for the smaller water craft where passive inspections are most valuable. In this project we performed tests and improved the performance of an instrument (developed earlier under, "Motion Correction for Ship-to-Ship Passive Inspections") that uses automated tracking of a target vessel in visible-light images to generate a 3D radiation map of the target vessel from data obtained using a gamma-ray imager.

\section{BACKGROUND}

When considering motion between two vessels one has to contend with nine relative degrees of freedom: the roll, pitch, and yaw of each vessel plus their relative separation (x, y, z). This relative motion keeps one from applying radiation imaging to the ship-to-ship detection problem since the apparent location of a source will wander through the image. This will significantly reduce the sensitivity of the data obtained. However, if one can determine the relative orientation and location of the target vessel in the radiation camera's field of view, then one can compensate for the motion and retain the advantages of imaging. This approach has been successfully used to enhance the detection of sources in moving vehicles where a large gamma-ray imager parked next to a multilane roadway can uniquely identify the radiation signature from each of the passing vehicles [1]. In this, and a prior project we have developed the tools that allow us to compensate for the much more complicated inter-vessel motion.

The project leverages earlier work where we first measured the relative motion expected for small vessel-to-vessel radiation inspections. Using this information together with concepts of operations for inspections, we developed suitable hardware for such inspections. That hardware was deployed on the water for the first time early in FY13. For the remainder of the year we have worked to analyze that data and improve the performance of the system.

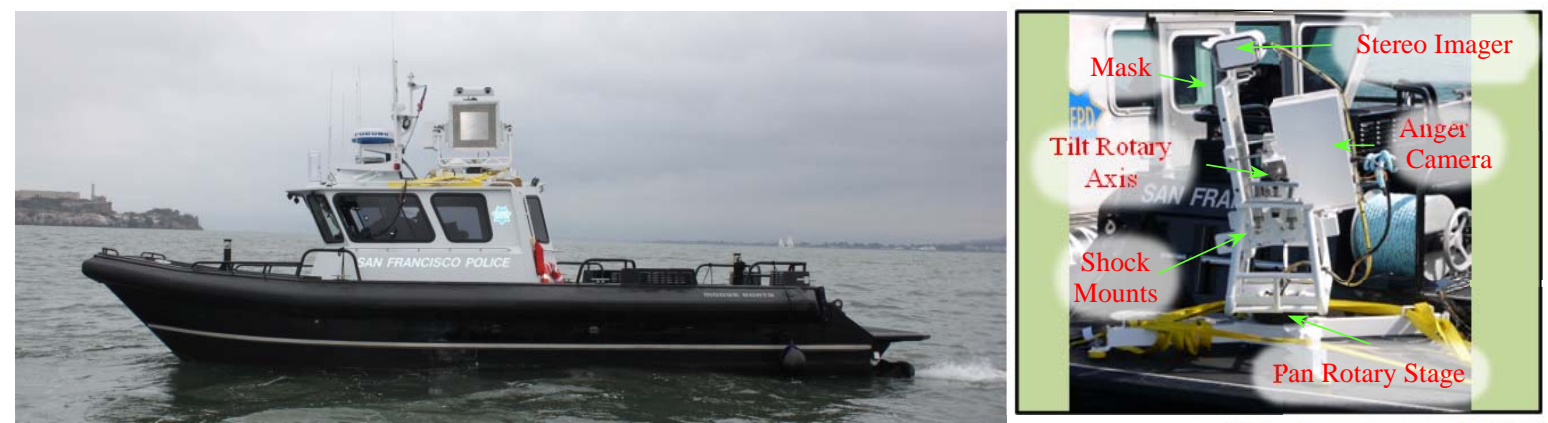

Fig. 1. Ship-to-ship imager mounted to the roof of a SFPD patrol vessel (left). Side view of the instrument (right.) 


\section{EXPERIMENTAL RESULTS}

The instrument developed to perform the ship-to-ship inspections is shown mounted on a San Francisco Police Department patrol vessel in Fig. 1. It comprises a custom Anger Camera-based, coded-aperture, gamma-ray imager, mounted on a two-axis gimbal. A stereo visible-light imager is mounted to, and aligned with the gamma-ray imager. The motorized gimbal is designed only to keep the target vessel in the system field of view. Precise motion correction is handled digitally by the various software components.

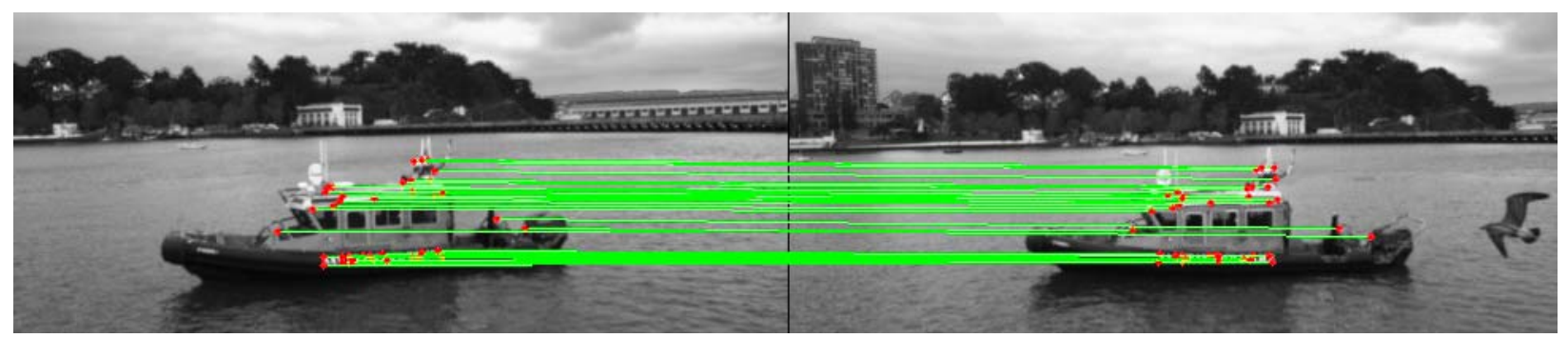

Fig. 2. Images of a target vessel showing the correspondence between points found by the tracking system.

The software suite comprises several modules, including a user interface, gimbal control, gamma-ray image generation, Tomography generation, and target tracking. At the heart of the system is the visual odometry module. This receives the visible light and discrepancy (distance) images from the stereo imager and uses routines from the OpenCV (Open Source Computer Vision) library [2] to perform its task of determining the location and orientation of the imaging system. The first step on receiving a video image is to find "Good features to track [3]". This finds locations in the 2D image where there are strong gradients in more than one direction. Such points are generally unique and can be found in subsequent frames (Fig. 2). Once a set of points has been found, the software finds the distance to those points using the disparity image. After this step the system has a series of 3D points and these are used to define a coordinate system attached to the target vessel. The last step in processing a frame is to calculate the location and orientation of the video camera in the 3D coordinate frame determined above.
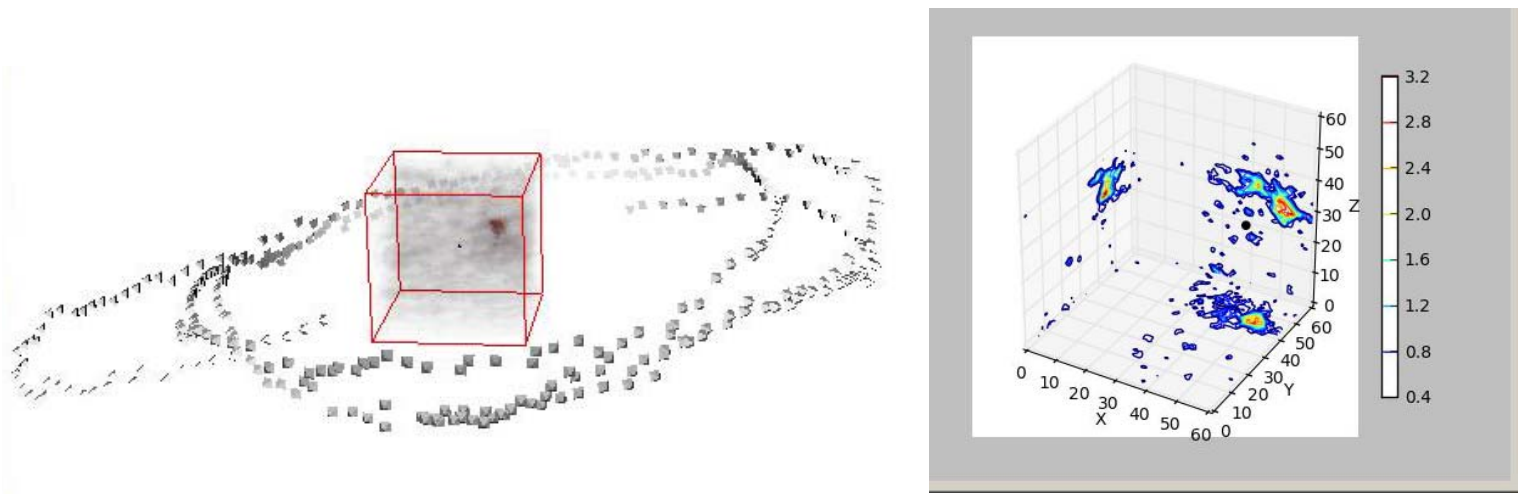

Fig. 3. Left: Track and pointing of the gamma-ray imager around the target volume (red cube). Each of the gray "points" along the track is a pyramid opening in the direction the system is looking. A source is visible in the upper right of the cube. Right: Source intensity projected onto the three coordinate planes. A 50\% threshold is applied.

When the next video image comes in, the software goes through the same steps to find points and assign them a 3D location. In addition, optical flow routines are applied to identify points that were used in the prior image (Fig. 2). Using the common set of 3D points between the two images, a landmark transform is used to determine the 4D transformation matrix that takes the coordi- 
nate system in the first image to the new orientation in the current image. With this information the system then calculates the new location and orientation of the imagers.

The gamma-ray imager collects data for the time between video frames, and uses that data to make a short exposure gamma-ray image. Using the orientation information from the video analysis, the image is then projected back onto the voxels of a cubic volume centered on the target vessel. If a source is present, over time it will show up in the target volume (Fig. 3).

\section{RESULTS, DISCUSSION AND CONCLUSIONS}

The results of this initial exercise showed that the system worked. However, they also revealed that the source detection was not as significant as one might expect. This was due to cumulative errors in the target tracking. In fact, as the inspecting vessel circled the target vessel, the visual odometry module systematically underestimated the number of degrees traveled around the target by 10-30\%. This is not unexpected for the results of a first field trial for a new instrument, and the remainder of the year was spent in improving system performance. Recognizing that such work would be required, the field trial data were all collected in list mode. Hence, they could be replayed as if the system were in the field.

Software improvements focused on two issues. First, for any frame the software would mismatch a few points or use points not on the target vessel. These would add an error to the landmark transform, providing an error in the motion between the two 3D coordinate systems. To improve this, after the landmark transform, we filtered outlying points (those that did not retain a reasonable 3D position with respect to the other points), and then performed the transform a second time. Second, even after this fix, each transform will have a small error and if one builds the host vessel's track frame by frame, then the "stack-up" error will become significant. To minimize this, the software uses "reference" frames. At any instant the relative orientations are determined with respect to the last reference frame. Only when the aspect of the target has changed so much that the system cannot find correspondence of a sufficient number of points between the current frame and the last reference frame is a new reference frame chosen. The transforms between reference frames are used to determine the global system track. Overall these changes have significantly improved performance, but we are still studying how robust the changes are, i.e. we do not want parameters that only work for a particular run.

\section{PATH FORWARD}

We are exploring the errors and stability of the improved tracking parameters and various software changes and these will be used to write a paper on the project results.

\section{PRESENTATIONS AND PUBLICATIONS}

- An oral presentation on the system was presented at the 2012 IEEE Nuclear Science Symposium.

- A paper to IEEE Transactions of Nuclear Science is in preparation.

\section{REFERENCES}

1. K.P. Ziock, et al., "Performance of the Roadside Tracker portal-less portal monitor,” IEEE Trans. Nucl. Sci., vol. 60, pp. 2237-2246, 2013.

2. Available from http://sourceforge.net/projects/opencvlibrary/.

3. J. Shi, C. Tomasi, “Good features to track,” Proc. Computer Vision and Pattern Recognition'94 IEEE Comp. Soc. Conf. pp., 593-600, 1994. 